\title{
DEVELOPMENT OF A PRECISION AMPLIFIER FOR THE DETECTOR*
}

\author{
K. H. Park ${ }^{\#}$, S. H. Jeong, S. M. Hong, W. W. Lee, Y. G. Jung, D. E. Kim, H. S. Lee, C. W. Chung, \\ PAL, POSTECH, Pohang, Korea \\ B. K. Kang, Department of Electrical Engineering, POSTECH, Pohang, Korea
}

\begin{abstract}
A trans-resistance amplifier has been designed and tested to measure the intensity of synchrotron radiation at Pohang Light Source (PLS). The front-end stage of the amplifier was implemented using discrete circuit elements and can measure input current in the range of $1 \mathrm{pA}$ to $1 \mu \mathrm{A}$. A microprocessor interfaces the amplifier with computer and controls the other sub-circuits for synchrotron radiation measurement. The amplifier was tested and obtained a resolution of $\sim 20 \mathrm{fA}$, a temperature drift of $\sim 0.1 \mathrm{pA} /{ }^{\circ} \mathrm{C}$, and a signal-to-noise ratio greater than $80 \mathrm{~dB}$.
\end{abstract}

\section{INTRODUCTION}

Commercial operational amplifiers such as the OPA128 from Burr-Brown Co. and the LMC6001 from National Semiconductor Co. have been used as a building block for a trans-resistance amplifier in $\mathrm{nA}$ range. When we use the commercial amplifiers for the synchrotron radiation detector, the circuit becomes very simple but it is difficult to achieve the desired properties for the detector. A transresistance amplifier was designed to measure the intensity of the incoming synchrotron radiation to the beam-line of PLS. It uses a cascode differential amplifier (CDA) to sense an ultra low input current [1]. This CDA has been build using $\mathrm{n}$-channel junction field-effect transistor (JFET) [2-3] and it works as a front-end stage of the preamplifier for the detector. The output of CDA has been amplified using a commercial operational amplifier. The synchrotron radiation detector system for PLS, the design of trans-resistance amplifier, and various test results of the amplifier are given in this paper.

\section{DETECTOR SYSTEM}

A block diagram of the synchrotron radiation detector is shown in Fig. 1. It consists of an ion-chamber, CPU module, high voltage (HV) supply, precision amplifier, voltage to frequency $(\mathrm{V} / \mathrm{F})$ converter, etc. The CPU module was implemented using a C8051F060 microcontroller from Cygnal Co., which has analog/digital peripherals and on-chip RAM. One of the two ADCs on C8051F060 micro-controller measures the current and the other measures the high voltage output. The DAC output of C8051F060 micro-controller controls the set voltage for HV supply. The ion chamber was biased by the HV supply with variable voltage from 0.15 to $1.5 \mathrm{kV}$. The amplifier of a trans-resistance type measures the output current of ion chamber. The gain of this amplifier can be selected from $10^{6}$ to $10^{12} \mathrm{~V} / \mathrm{A}$ at a step of $10^{i}$, where $i=0,2 \ldots 6$, using the 9011 miniature reed relays from COTO Technology. The 9011 miniature reed relay has an insulation resistance $>1 \mathrm{~T} \Omega$. An AD650 voltage to frequency $(\mathrm{V} / \mathrm{F})$ converter from Analog Devices Co. converts the output voltage of amplifier for an external frequency counter. The error on $\mathrm{V} / \mathrm{F}$ conversion was $\sim 0.01 \%$. The interface between the computer and the CPU of system was made through the RS232C interface.

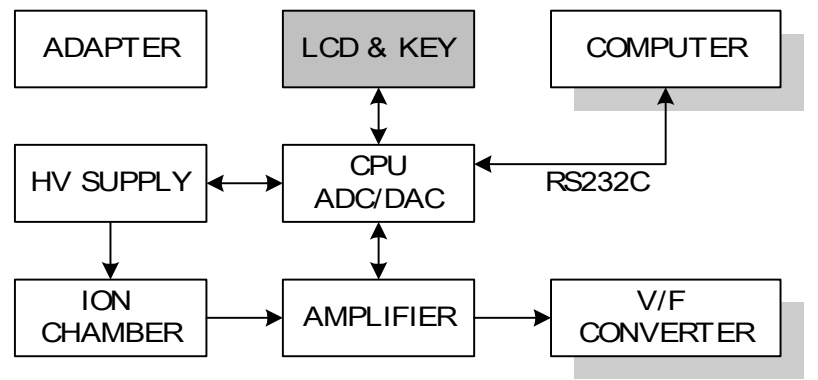

Figure 1: A block diagram of the synchrotron radiation detector system.

\section{TRANS-RESISTANCE AMPLIFIER}

A trans-resistance amplifier inputs a current and outputs a voltage. An ideal trans-resistance amplifier has an input and output resistances of $0 \Omega$ as shown in Fig 2 .

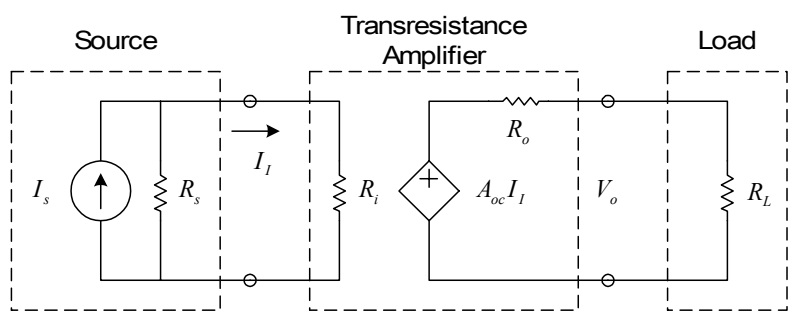

Figure 2: Model for the trans-resistance amplifier.

The trans-resistance amplifier designed for the synchrotron radiation detector is shown in Fig. 3. It uses a CDA as a front-end stage. The CDA has the following advantages; it keeps the drain-to-gate voltage $V_{D G}$ low and prevents breakdown of JFET. The input conductance of CDA is reduced to $g_{i}=g_{g s 1}+2 g_{g d 1}$ because of the Miller effect, and the gate leakage current of the input JFET is minimized. Here, $g_{g s 1}$ and $g_{g d 1}$ are the gatesource and gate-drain conductances of the input JFET. 


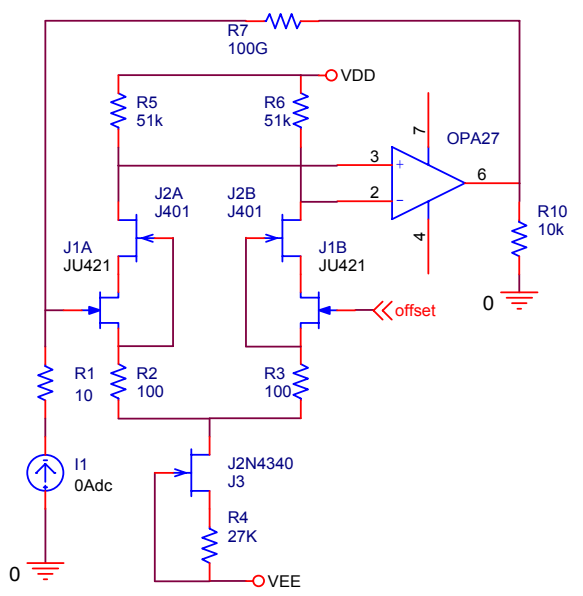

Figure 3: A circuit diagram of the trans-resistance preamplifier.

The CDA was implemented using a JU421 dual JFET from Vishay Inc. because it has a low gate leakage current. The specified gate leakage current of JU421 is $0.2 \mathrm{pA}$ in maximum at $V_{D G}=10 \mathrm{~V}$ and $I_{D}=30 \mu \mathrm{A}$. The input resistance $R_{i}$ of the implemented CDA is $\sim 3.4 \mathrm{~T} \Omega$. The gain of CDA is defined as $A_{v c}=\left(v_{d 1}-v_{d 2}\right) / v_{i d}=-g_{m 1} R_{L}$. Because $g_{m 1}=0.3 \mathrm{mS}$ for JU421 and $R_{L}=51 \mathrm{k} \Omega$, the CDA has a voltage gain of $\sim 23 \mathrm{~dB}$. The open loop gain of the operational amplifier OPA27 is $117 \mathrm{~dB}$. Thus the two-stage preamplifier provides a total open-loop gain $A_{O L}$ of $\sim 140 \mathrm{~dB}$. It is very close to the simulated value of $146 \mathrm{~dB}$ using PSPICE. A feedback resistor R7 provides a shuntshunt feedback. In a shunt-shunt feedback circuit, the input resistance of the amplifier $Z_{i}$ is $\left(R_{F} / / R_{i}\right) /(1+T)$, where $T$ is the loop gain, $R_{i}$ is input resistance of the amplifier, and $R_{F}$ is the feedback resistor, respectively. The loop gain $T$ is close to $A_{O L}$ if $R_{i} \geq R_{F}$. When $R_{i}=3.4 \mathrm{~T} \Omega$ and $R_{F}=0.1 \mathrm{~T} \Omega, Z_{i}$ becomes $\sim 10 \mathrm{k} \Omega$, which is much smaller than the output resistance of the ion chamber. The output resistance of the ion chamber is very high like open circuits.

\section{NOISE ANALYSIS}

A noise model for the preamplifier is given in Fig. 4. The ion chamber is represented in Fig. 4 with a parallel connection of a current source $I_{D}$, resistor $R_{D}$, and capacitor $C_{D}$. The noise sources $i_{n i}$ and $e_{n i}$ represent the noise current and voltage at the inverting input terminal of the amplifier, and the capacitor $C_{i a}$ represents the input capacitance. The noise voltage of the feedback resistor $R_{F}$ is represented with the noise voltage source $e_{n R}$. The parasitic capacitance of the feedback resistor is represented with $C_{S}$.

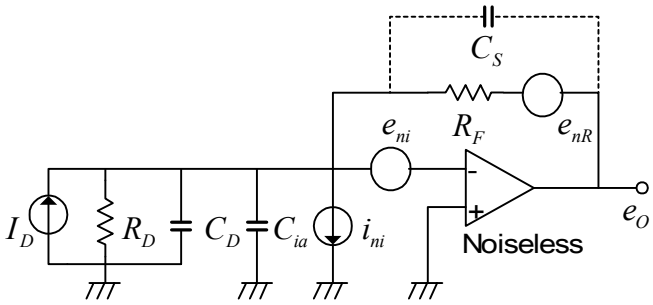

Figure 4: A noise model for the trans-resistance preamplifier.

The spectral noise density of the feedback resistor $R_{F}$ is given as $e_{n R}=\sqrt{4 k T R_{F}}$ and the noise voltage source $e_{n R}$ is $\sim 40 \mu \mathrm{V} / \sqrt{\mathrm{Hz}}$ when $T=298{ }^{\circ} \mathrm{K}$ and $R_{F}=100 \mathrm{G} \Omega$. Here, $k=1.38 \times 10^{-23}$ is the Boltzman's constant. The noise current $i_{n i}$ due to a gate leakage current $I_{B-}$ is given as $i_{n i}=\sqrt{2 q I_{B-}}$, where $q=1.602 \times 10^{-19} \mathrm{C}$ is the charge of electron. For JU421, $I_{B-}$ is $\sim 0.2 \mathrm{pA}$ and the noise current $i_{n i}$ is $\sim 0.25 \mathrm{fA} / \sqrt{\mathrm{Hz}}$. Most of this noise current flows into the feedback resistor and results in an output noise voltage $e_{n R i}$ of $25 \mu \mathrm{V} / \sqrt{\mathrm{Hz}}$. For $C_{D}>>C_{S}$, the output noise density $e_{\text {noe }}$ due to the $e_{n i}$ is given by (1)

$$
e_{\text {noe }} \cong \frac{1+s R_{F}\left(C_{D}+C_{i a}\right)}{1+s R_{F}\left(C_{S}\right)} e_{n i}
$$

Because $C_{D} \cong 100 \mathrm{pF}$ for an ion chamber, and $C_{i a}=3$ $\mathrm{pF}$ for JU421, and $e_{n i} \approx 10 n \mathrm{~V} / \sqrt{\mathrm{Hz}}$ at a low frequency range, we have $e_{\text {noe }} \approx 4.0 \mu \mathrm{V} / \sqrt{\mathrm{Hz}}$, which is small compared to $e_{n R}$. Combining the above three noise sources, the rms output noise voltage becomes (2)

$$
e_{n o}=\sqrt{e_{n R}^{2}+e_{n R i}^{2}+e_{\text {noe }}^{2}} \cong 47 \mu \mathrm{V} / \sqrt{\mathrm{Hz}}
$$

The measured noise spectral density using the $35670 \mathrm{~A}$ dynamic analyzer from Agilent Co. is shown in Fig. 5.

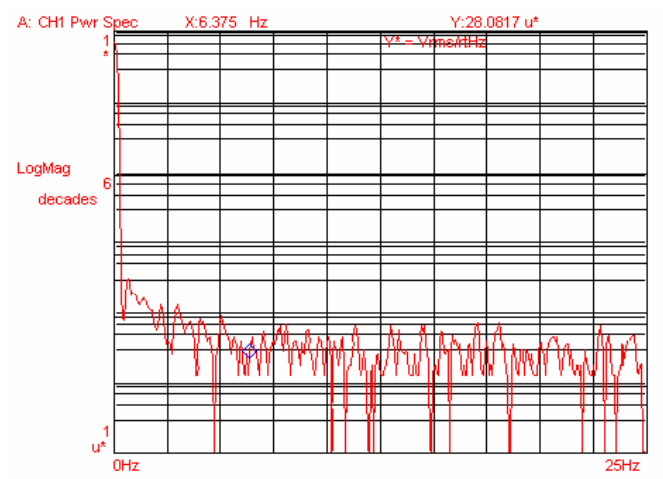

Figure 5: Noise spectral density of the trans-resistance preamplifier.

The measured noise level is $\sim 30 \mu \mathrm{V} / \sqrt{\mathrm{Hz}}$, which is close to the calculated one. 


\section{EXPERIMENTAL RESULTS}

The measured output voltage of amplifier and the measured frequency of $\mathrm{V} / \mathrm{F}$ converter for an input current of $1 \sim 10 \mathrm{pA}$ are in Fig. 6 . The input current was increased at a step of $1 \mathrm{pA}$ using the 6430 Sub-Femto Source meter from KEITHLEY Co. The results show that the measurement error is $\sim 0.03 \%$.

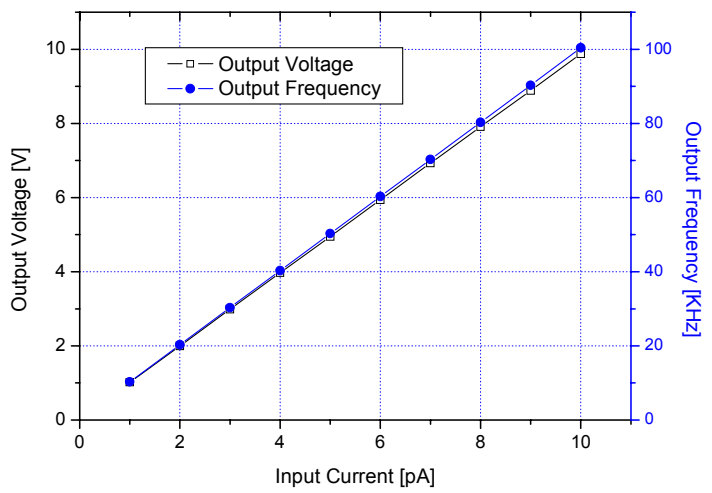

Figure 6: The output voltage of amplifier and the measured frequency of $\mathrm{V} / \mathrm{F}$ converter measured using an input current source.

The beam intensity of the $4 \mathrm{C} 1$ beam line at PLS was measured using the fabricated ion chamber and amplifier and the results are shown in Fig. 7. During a time interval of one hour, the measured photon beam current decreased linearly from 8.7 to $8.4 \mathrm{nA}$, while the electron beam current of the storage ring changed from 141 to $135 \mathrm{~mA}$. This result shows that the fabricated detector is appropriate for measuring the photon beam current.

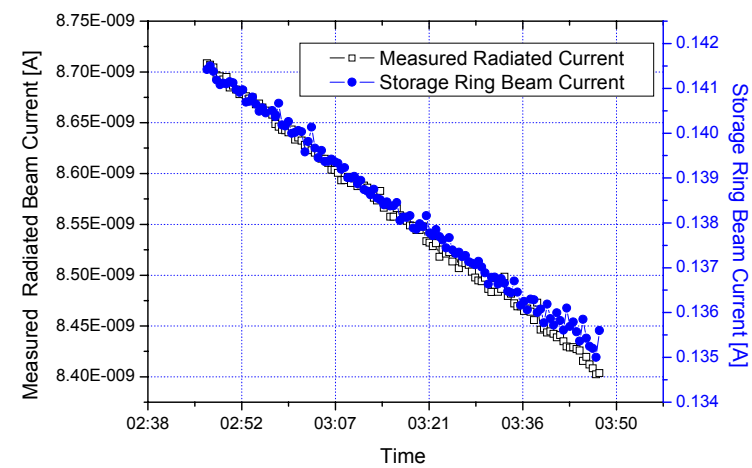

Figure 7: The measured photon beam currents of the 4C1 beam line and the measured electron beam current of the storage ring of PLS.

To check the variation of amplifier gain due to temperature drift, a constant input current in $\mathrm{pA}$ range was applied to the amplifier using the 6430 Sub-Femto Source meter and the measured output voltage was observed. The measured output voltages were converted into current values and the results are shown in Fig.8. This experiment was performed for a time period of 12 hours. The temperature change during the $12 \mathrm{hr}$ period was $\sim 1^{\circ} \mathrm{C}$. From the Fig. 8, one observes that the measurement error due to temperature drift is $\sim 0.1 \mathrm{pA} /{ }^{\circ} \mathrm{C}$ and the resolution for current measurement is better than $20 \mathrm{fA}$.

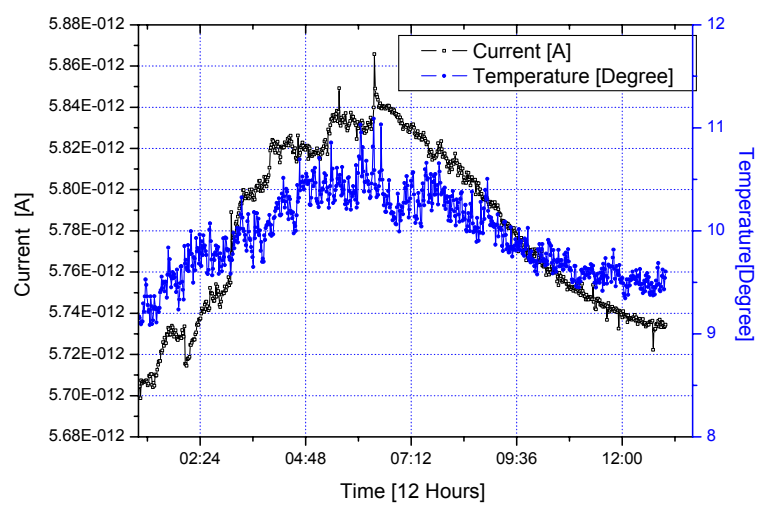

Figure 8: Amplifier drift due to a temperature variation.

\section{CONCLUSIONS}

A trans-resistance amplifier has been designed and tested to measure the intensity of synchrotron radiation at PLS. The front-end stage of the amplifier was implemented using discrete circuit elements. The amplifier has a resolution of $20 \mathrm{fA}$ for current measurement, a signal-to-noise ratio of $\sim 80 \mathrm{~dB}$, and a measurement error of $\sim 0.1 \mathrm{pA} /{ }^{\circ} \mathrm{C}$ due to temperature drift. When it was installed on the 4C1 beam line at PLS, the experimental results indicate that the amplifier is appropriate for measuring the beam intensity of beam line.

\section{REFERENCES}

[1] Michael J, et al., "A Low-Noise, Wide-Band CMOS Charge-sensitive Preamplifier for use with APD/LSO PET Detectors", IEEE Transactions on Nuclear Science, Vol. 43, No.3 June 1996. p. 1666-1671

[2] A. Fazzi, et al., "Charge-Sensitive Amplifier FrontEnd with an nJFET and a Forward-Biased Reset Diode", IEEE Transactions on Nuclear Science, Vol. 43, No.6, December 1996. p. 3218-3222

[3] V. Radeka, et al., "Monolithic preamplifier employing epitaxial n-channel JFETs", Nuclear Instruments and Methods in Physics Research A326 (1993) 77-81 